\title{
Infiltration of White-Spot-Lesions and developmental enamel defects
}

\author{
Michael Knösel ${ }^{1 *}$, Roberto Vogel ${ }^{2}$, Paulo Sandoval ${ }^{3}$
}

\section{Professor, Department of Orthodontics,}

University Medical Center Göttingen (UMG), Göttingen, Germany, Visiting Professor, Department of Paediatric and Orthodontics, Universidad de La Frontera (UFRO), Temuco, Chile, and Private Practice, Hamburg, Germany 2. Assistant Professor, Department of Orthodontics, Universidad de La Frontera (UFRO), Temuco, and Private Practice, Temuco, Chile

3. Professor, Department of Orthodontics, Universidad de La Frontera (UFRO), Temuco, and Private Practice, Temuco, Chile

* Corresponding author. Prof. Dr. Michael Knösel Lübecker Str. 128, 22087 Hamburg, Germany Email:mknoesel@yahoo.de - info@kfo.hamburg Financiado por Proyecto MEC-Conicyt $N^{\circ}$ 80130052

Trabajo recibido el 18/06/2016. Aprobado para su publicación el 08/02/2017

\begin{abstract}
Purpose: To provide an overview of different indications suitable for treatment with the technique of resin infiltration (Icon, DMG), such as white-spot lesions (WSL), enamel fluorosis, and molar-incisor hypomineralisation (MIH), and to propose a valid and strategy feasible in dental surgeries for screening of dental color improvement and -stability. Method: A non-systematic review of the literature was performed to characterise inhibition of lesion progression, and the esthetic effects induced by resin infiltration of decalcified enamel, enamel fluorosis, and molar-incisor hypomineralisation (MIH), using the online database Pubmed and a suitable search strategy. Database search was complemented by a hand-search of references of retrieved articles. Results: Of 34 retrieved articles, 29 were included in the review. Evidence of an assimilation of WSL color to surrounding enamel following infiltration is available on the level of RCTs, and the results were found to be stable in color with no significant changes over at least twelve months. Evidence of an esthetic improvement of fluorotic or MIH affected teeth is available on the level of case reports and case control studies. Conclusion: As a micro-invasive approach to infiltrate and seal different types of enamel lesions, with a subsequent improvement of the esthetic appearance, resin infiltration is considered to be a useful complementation of the dental therapeutic specter.
\end{abstract}

KEYWORDS

Review; White spot lesion; Fluorosis; MIH; Resin Infiltration; Icon

Rev. Clin. Periodoncia Implantol. Rehabil. Oral Vol. 10(2); 101-106, 2017.

\section{INTRODUCTION}

Orthodontic corrections are mostly achieved with fixed orthodontic appliances. One of the negative side-effects is the formation of white spot lesions (WSL) and incipient caries ${ }^{(1,2)}$. Prevention of whitespot lesions (WSL) during fixed appliances orthodontic treatment is still a challenge in today's orthodontic treatment: There is evidence that neglecting oral hygiene during orthodontic treatment with fixed appliances can cause WSL formation within weeks ${ }^{(3,4)}$. Orthodontic patients develop significantly more WSL than non-orthodontically treated persons, mainly because oral hygiene is more difficult in patients with multi-bracket orthodontics than without fixed treatment. Not treating WSL has been shown to result in lesion progression to carious lesions, and moreover presents esthetic problems ${ }^{(3)}$. WSL formation has been described as a rapid process of few weeks duration, with a sharp increase in WSL numbers within the first six months ${ }^{(4-6)}$. The incidence of WSL in individuals treated with fixed appliances has been reported to be $72.9 \%$ of patients, of which $2.3 \%$ of lesions were cavitated $^{(7)}$. Even inactive lesions which do not show a tendency to progress may constitute an esthetic problem in anterior teeth, and one that is often noticed by patients not during treatment, but following appliance removal. While in the case of cavitated lesions restoration is essential, non-cavitated lesions should receive preventive therapy aimed at arresting and remineralizing the lesion ${ }^{(8)}$. As a new way of inhibiting further demineralization and improving their appearance, lesion infiltration with low-viscosity light-curing resins (infiltrants) has been recently proposed ${ }^{(9)}$. A reduced visibility of infiltrated WSlesions is an additional positive side-effect, which is due to the similar refractive index of the infiltrated and sound enamel areas. Other enamel alterations such as further enamel anomalies such as molarincisor-hypomineralisation $(\mathrm{MIH})$, or fluorotic enamel also require esthetic improvement ${ }^{(10-13)}$. It has been reported that beyond the aspect of treating post-orthodontic WSL, resin infiltration might be suitable for treating those enamel anomalies (Figure 1).

\section{Aim of the review}

It is the objective of this review to provide an overview of different

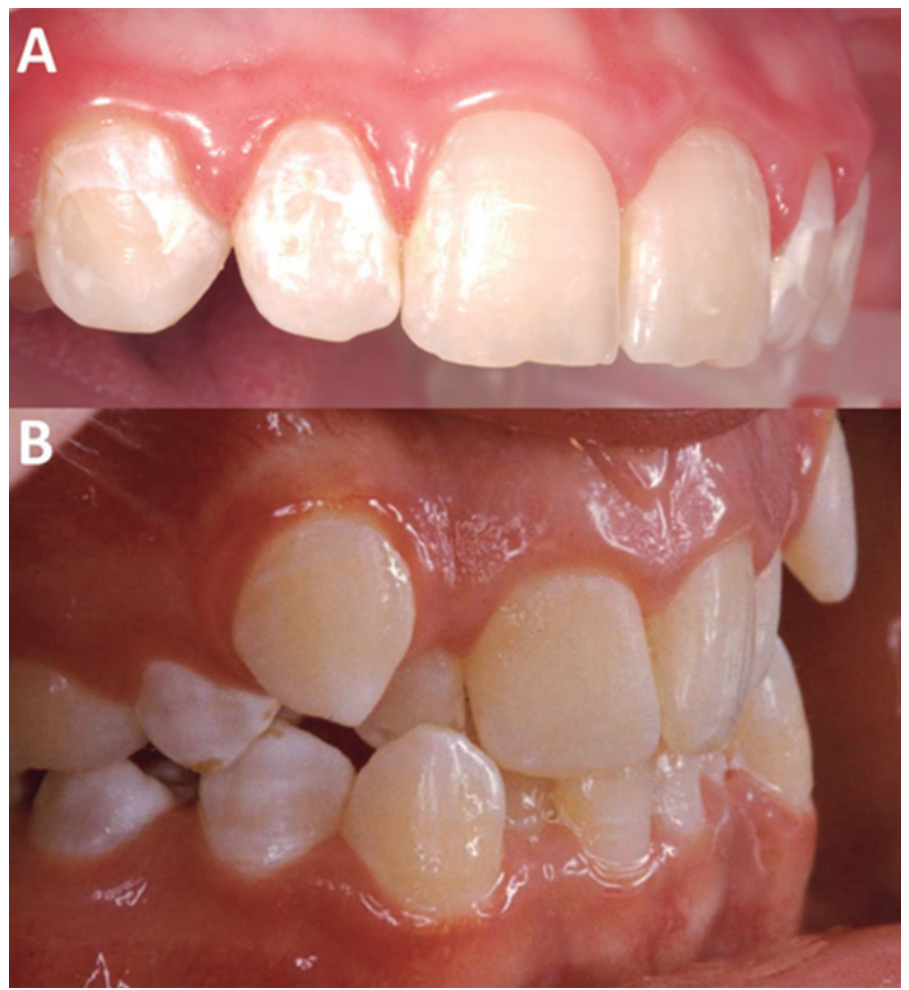

Figure 1. Examples of post-orthodontic WSL (A), and dental fluorosis (B).

indications suitable for treatment with the technique of resin infiltration (Icon, DMG), such as white-spot lesions (WSL), enamel fluorosis, and molar-incisor hypomineralisation (MIH). 


\section{METHOD}

\section{Search strategy}

A non-systematic review of the literature using the PubMed database and robust truncations was performed on Feb 16, 2016 with no restrictions, to characterise inhibition of lesion progression, and the esthetic effects induced by resin infiltration of decalcified enamel, enamel fluorosis, and molar-incisor hypomineralisation $(\mathrm{MIH})$. The search terms used in the PubMed database were

(infiltration) OR (Icon) OR (micro-invasive) AND ("white-spot" OR "white spot" OR "decalcified" OR "decalcification" OR "caries" OR "MIH" OR "hypomineralisation" OR "fluorosis" OR "fluorotic") AND ("esthetic" OR "camouflage" OR "mask" OR "conceil*”)

Database search was complemented by a hand-search of references of retrieved articles.

\section{RESULTS}

PubMed database search yielded 21 results. Hand-search of references of retrieved articles yielded another 13 articles. Five articles were not considered as they were not related to the topic of esthetic improvement of WSL, MIH or fluorosis affected enamel appearance by resin infiltration. Table 1 gives an overview of the articles retrieved by Pubmed, and by additional hand search of references.

\section{DISCUSSION}

\section{Post-orthodontic WSL in vitro}

The positive effects of masking vestibular white spots as well as the color stability of infiltrated lesions have been quantified in vitro by several research teams ${ }^{(9,14-18)}$. Additionally, a current costeffectiveness analysis indicates that non-invasive treatment of early WSL by infiltrating instead of or postponing restorative treatment can have an impact on improving oral health care as it lengthens the lifecycle of a tooth ${ }^{(19)}$. The surfaces of infiltrated lesions are described as protected enamel surfaces following appropriate polishing, in vitro $^{(20,21)}$. These finding is underlined by the description of an increase of mechanical strength after resin infiltration by other authors ${ }^{(22-24)}$.

Infiltrated lesions seem to be protected against new acid induced demineralization, and based on different in vitro experiments, a combination of resin infiltration and several adhesive systems seems to be viable, thereby allowing for a proceeding with fixed orthodontic treatment following an infiltration intervention ${ }^{(22,25,26)}$. Even more, there seems to be an enhanced performance of adhesives compared to untreated demineralized enamel: Some research groups quantified the bond strength of orthodontic brackets to infiltrated enamel ${ }^{(16,27-30)}$ and found that a mechanical inforcement of infiltrated surfaces seems to reduce enamel cracks or surface damages after debonding, in vitro ${ }^{(29)}$. As for the masking effect, de Lacerda et al. found that the Icon infiltrant produced a greater lightness reduction of artificial white-spot lesions in vitro, when compared to other sealants. $\mathrm{Ou}$ et al. reported that an excellent masking effect, too, with a high color stability, and no differences in terms of the masking effect with treatment of high or low demineralized enamel. Yim et al. evaluated removed surface layers and infiltrated areas of human enamel surface thicknesses in vitro, using confocal laser scanning microscopy, and found that application of $37 \% \mathrm{H} 3 \mathrm{PO} 4$ with a brush for 30 seconds increased the pore volume of WSL surface layers and the percentage of infiltrated areas in comparison to the use of $15 \% \mathrm{HCl}$ for 120 seconds. Cohen-Carneiro et al. used bovine enamel specimens to test the discoloration potential of different agents (saliva, coffee, and wine) of infiltrated and non-infiltrated WSL. They used very long immersion intervals and found that infiltrated WSL had a higher staining potential than non-infiltrated WSL, in vitro. In contrast, Paris found also in vitro that resin infiltration is suitable to mask artificial WSL, and that polished infiltrated lesions are resistant to staining in vitro. ${ }^{(9)}$

Yuan et al. compared the esthetic improvements of white-spot lesions (WSLs) treated by fluoride, casein phosphopeptide amorphous calcium phosphate (CPP-ACP), or resin infiltration in vitro, and found that resin infiltration was more effective than $\mathrm{NaF}$ or CPP-ACP in masking WSLs. Paris and Meyer-Lueckel described the potential of the infiltration technique in dental practice ${ }^{(31)}$ as an intervention that bridges non-invasive and restorative treatment options, by arresting and masking non-cavitated proximal caries lesions.

\section{Adverse effects on WSL or sound enamel}

Up until now, no adverse effects are reported for the use of WSL infiltration. The technique has been used in clinical application with excellent results inmicro-invasive treatment of early approximal / vestibular caries). During infiltration treatment, the sound enamel adjacent to the WSL is likewise treated; i.e., both areas, sound enamel and WSL, are etched with $\mathrm{HCl}$ gel and treated with the infiltrant. However, according to a recent $\mathrm{RCT}^{(32)}$ no adverse effects were seen following etching of sound enamel along with WSL infiltration. This is supported by a previous in-vitro research: Meyer-Lueckel et al. found no significant differences in erosion depths following etching between lesions and sound enamel(33). Etching and subsequent infiltration of sound enamel does not alter its structure too much, and the etching removes more of the porous WSL enamel than of the sound enamel.

\section{Post-orthodontic WSL in vivo}

Several studies have been dedicated to the masking effects of post-orthodontic WSL by infiltration ${ }^{(9,34,35)}$. The effect of lesion progression as seen in approximal lesions was confirmed for labial lesions, also ${ }^{(34,36)}$. Furthermore, case reports and short-term studies $^{(9,32,34,35,37-45)}$ describe a masking of WSL, up until 1 year following infiltration ${ }^{(32,37,46)}$.

Hammad et al. distinguished between infiltration of visible WSLs without surface disruption and WSLs with a roughened surface not requiring restoration, and reported significant improvement in both groups following infiltration. Likewise, Shivanna and Shivakumar, as well as Kugel et al. presented a case reports along with reviews on the subject of the infiltration technique, and characterised it as a significant addition to clinician's caries.

Using an RCT design, Senestrao et al. noted a marked improvement of decalcified enamel following infiltration in comparison to control teeth, with color stable results eight weeks after treatment. Another recent long-term $\mathrm{RCT}^{(32)}$ assessed the assimilation of infiltrated WSL with surrounding sound enamel. Similar to Senestrao et al., these authors found an assimilation of WSL color to surrounding enamel following infiltration that was color stable, without significant changes over six months. The extent of assimilation was influenced by extent and depth of lesions, and lesion surfaces: the more superficial and ,younger' the lesion, the better the masking effect. The longer the time elapse between debonding and infiltration, the more etching intervals are needed to achieve camouflage effects, which are also less satisfying. In a follow-up study, the same authors assessed the stability of the results, again: Esthetic results remained stable of at least twelve months of observation. Further studies are required to assess the potential limits of the durability of infiltration. Adverse effects in terms of gingiva irritation or pain were assessed but not seen by this trial.
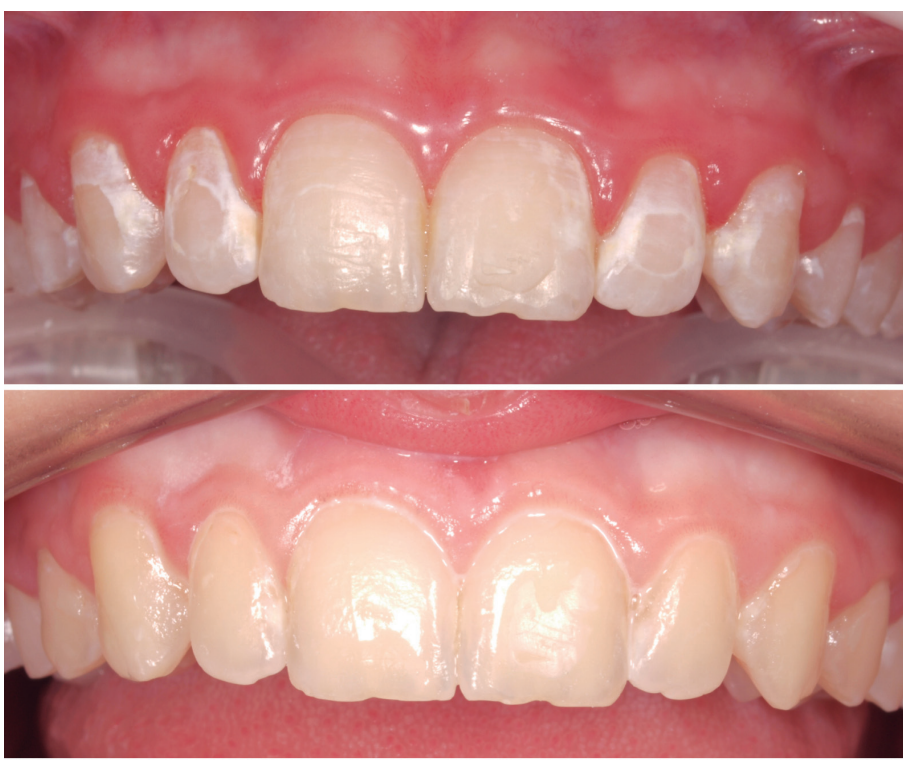

Figure 2. Infiltration of post-orthodontic WSL in upper front teeth: Prior to (above), and following infiltration (below). 


\section{MIH-, fluorosis affected teeth}

Infiltration of carious lesion has been characterised as a strategy to strengthen damaged enamel structures, and to reduce caries progression without surgical intervention ${ }^{(31,47-50)}$. Beyond the aspect of WSL and early caries treatment, several other investigators also described an esthetical improvement and mechanical stabilization of fluorotic teeth, traumatic white spots or teeth suffering from molar incisior hypomineralisation $(\mathrm{MIH})$ by infiltration, which increases its use as a therapeutic alternative for esthetic purposes in different types of porous lesions ${ }^{(51-56)}$. E.g., Kim et al. ${ }^{(41)}$ described the effects of infiltration on teeth with developmental enamel defect and post-orthodontic WSL. They found that $25 \%$ of developmental enamel defect were completely masked, whereas 35\% and $40 \%$ were partially masked and unchanged, respectively. $61 \%$ of WSL teeth were completely masked, $33 \%$ partially masked, and $6 \%$ unchanged. Munoz et al. (2013) described in a case report the promising results and of the infiltration technique for a microinvasive type of treatment in cases of mild-to-moderate fluorosis and hypoplasia stains. Likewise, Tirlet et al. report cases of fluorosis and traumatic hypo-mineralization lesions successfully treated by infiltration, thereby suggesting to extend the indication of the technique to those enamel alterations. In this sense, also Attal et al. provided a protocol to treat MIH affected enamel, deep lesions of traumatic origin, or those associated with fluorosis succesfully using the infiltration technique ${ }^{(51)}$. This was confirmed by Auschill et al (2014) who used the infiltration technique on cases of white opaque and brown discolourations by dental fluorosis of moderate extent, following experiencing unsatisfying results of bleaching to improve the aesthetic appearance of the affected teeth. The treatment results achieved by resin infiltration were satisfying in contrast, and remained stable in terms of a camouflage when assessed six months after treatment, the remain masked.

\section{CONCLUSIONS}

-WSL infiltration is a micro-invasive treatment option to treat decacified enamel and to inhibit further demineralization.

- Both RCTs, case reports and in vitro research indicate a reduction of visibility of infiltrated WSL, which is due to the similar refractive index of the infiltrant and sound enamel areas.

- The durability of the camouflage effect following infiltration has been shown to last for at least six months on the evidence level of RCTs, and at least one year according to a follow-up study of cases included in an RCT.

- Duration and depth of WSL are co-factors that affect the extent of improvement of the esthetic appearance of demineralised teeth by infiltration.

Table 1. Overview of the articles retrieved by Pubmed, and by additional hand search of references. Of 34 retrieved articles, 29 were included in the review.

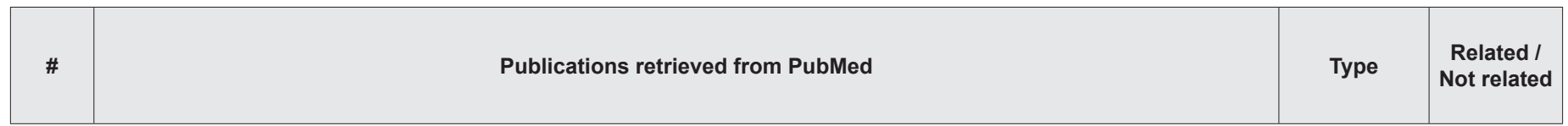

de Lacerda AJ, Ávila DM, Borges AB, Pucci CR, Torres CR. Adhesive Systems as an Alternative Material for Color Masking of White
Spot Lesions: Do They Work? J Adhes Dent. 2016 Jan 26. doi: 10.3290/j.jad a35518. [Epub ahead of print] PubMed PMID: 26814318.

In vitro,

enamel Genet Mol Res. 2014;13(3):6912-9. doi: 10.4238/2014.August.29.14. PubMed PMID: 25177972.

In vitro

Torres CR, Borges AB. Color masking of developmental enamel defects: a case series. Oper Dent. 2015;40(1):25-33. do: 10.2341/13-346-T. Epub 2014 Aug 19. PubMed PMID: 25136905.

case series

Yim HK, Min JH, Kwon HK, Kim BI. Modification of surface pretreatment of white spot lesions to improve the safety and efficacy

4 of resin infiltration. Korean J Orthod. 2014;44(4):195-202. doi: 10.4041/kjod.2014.44.4.195. Epub 2014 Jul 24. PubMed PMID:

In vitro,

of resin infiltration. Korean J Orthod. 2014;44(4):195-202. doi: 10.4041/kjod.2014.44.4.195.
25133134; PubMed Central PMCID: PMC4130915.

bovine

ename

follow-up of 5 Eckstein A, Helms HJ, Knösel M. Camouflage effects following resin infiltration of postorthodontic white-spot lesions in vivo: One-year follow-up of
follow-up. Angle Orthod. 2015;85(3):374-80. doi: 10.2319/050914-334.1. Epub 2014 Aug 15. PubMed PMID: 25126675.

Gugnani N, Pandit IK, Goyal V, Gugnani S, Sharma J, Dogra S. Esthetic improvement of white spot lesions and non-pitted fluorosis

6 using resin infiltration technique: series of four clinical cases. J Indian Soc Pedod Prev Dent. 2014;32(2):176-80. doi: 10.4103/09704388.130996. PubMed PMID: 24739922.

$7 \quad$ Yim HK, Kwon HK, Kim BI. Modification of surface pre-treatment for resin infiltration to mask natural white spot lesions. J Dent. 2014;42(5):588-94. doi: 10.1016/j.jdent.2014.01.015. Epub 2014 Feb 6. PubMed PMID: 24508542.

Case series

Cohen-Carneiro F, Pascareli AM, Christino MR, Vale HF, Pontes DG. Color stability of carious incipient lesions located in enamel and

8 treated with resin infiltration or remineralization. Int J Paediatr Dent. 2014;24(4):277-85. doi: 10.1111/ipd.12071. Epub 2013 Oct 17.

in vitro PubMed PMID: 24134448

bovine

Lee JH, Kim DG, Park CJ, Cho LR. Minimally invasive treatment for esthetic enhancement of white spot lesion in 9 adjacent tooth. J Adv Prosthodont. 2013 ;5(3):359-63. doi: 10.4047/jap.2013.5.3.359. Epub 2013 Aug 31. PubMed PMID:

Case report 24049579; PubMed Central PMCID: PMC3774952.

Yuan H, Li J, Chen L, Cheng L, Cannon RD, Mei L. Esthetic comparison of white-spot lesion treatment modalities

in vitro

human 
Table 1. -CONTINUATION- Overview of the articles retrieved by Pubmed, and by additional hand search of references. Of 34 retrieved articles, 29 were included in the review.

\begin{tabular}{|c|c|c|c|}
\hline 12 & $\begin{array}{l}\text { Knösel M, Eckstein A, Helms HJ. Durability of esthetic improvement following Icon resin infiltration of multibracket- } \\
\text { induced white spot lesions compared with no therapy over } 6 \text { months: a single-center, split-mouth, randomized clinical } \\
\text { trial. Am J Orthod Dentofacial Orthop. 2013;144(1):86-96. doi: 10.1016/j.ajodo.2013.02.029. PubMed PMID: } 23810050 \text {. }\end{array}$ & $\mathrm{RCT}$ & \\
\hline 13 & $\begin{array}{l}\text { Paris S, Schwendicke F, Keltsch J, Dörfer C, Meyer-Lueckel H. Masking of white spot lesions by resin infiltration in vitro. } \\
\text { J Dent. 2013;41 Suppl 5:e28-34. doi: 10.1016/j.jdent.2013.04.003. Epub 2013 Apr 11. PubMed PMID: } 23583919 .\end{array}$ & In vitro & \\
\hline 14 & $\begin{array}{c}\text { Paris S, Meyer-Lueckel H. The potential for resin infiltration technique in dental practice. Dent Update. 2012;39(9):623-6, } \\
\text { 628. PubMed PMID: } 23479851 .\end{array}$ & $\begin{array}{l}\text { Editorial / } \\
\text { letter }\end{array}$ & \\
\hline 15 & $\begin{array}{l}\text { Ontiveros JC. Commentary: alternative esthetic management of fluorosis and hypoplasia stains: blending effect obtained } \\
\text { with resin infiltration techniques(1). J Esthet Restor Dent. 2013;25(1):40-1. doi: 10.1111/j.1708-240.2012.00528.x. Epub } \\
2012 \text { Jul 10. PubMed PMID: } 23374408 .\end{array}$ & Letter & NR \\
\hline 16 & $\begin{array}{l}\text { Muñoz MA, Arana-Gordillo LA, Gomes GM, Gomes OM, Bombarda NH, Reis A, Loguercio AD. Alternative esthetic } \\
\text { management of fluorosis and hypoplasia stains: blending effect obtained with resin infiltration techniques. J Esthet Restor } \\
\text { Dent. 2013;25(1):32-9. doi: 10.1111/j.1708-8240.2012.00527.x. Epub } 2012 \text { Jul 10. PubMed PMID: } 23374407 \text {. }\end{array}$ & Case report & \\
\hline 17 & $\begin{array}{l}\text { Vailati F, Gruetter L, Belser UC. Adhesively restored anterior maxillary dentitions affected by severe erosion: up to 6-year } \\
\text { results of a prospective clinical study. Eur J Esthet Dent. 2013;8(4):506-30. PubMed PMID: } 24624375 .\end{array}$ & $\begin{array}{l}\text { case series } \\
\text { study }\end{array}$ & NR \\
\hline 18 & $\begin{array}{l}\text { Gugnani N, Pandit IK, Gupta M, Josan R. Caries infiltration of noncavitated white spot lesions: A novel approach for } \\
\text { immediate esthetic improvement. Contemp Clin Dent. 2012;3(Suppl 2):S199-202. doi: 10.4103/0976-237X.101092. } \\
\text { PubMed PMID: 23230363; PubMed Central PMCID: PMC3514922. }\end{array}$ & Case report & \\
\hline
\end{tabular}

19

Wang Y, Sa Y, Liang S, Jiang T. Minimally invasive treatment for esthetic management of severe dental fluorosis: a case report. Oper Dent. 2013;38(4):358-62. doi: 10.2341/12-238-S. Epub 2012 Dec 4. PubMed PMID: 23210860.
Case report

Case report
20

Kugel G, Arsenault P, Papas A. Treatment modalities for caries management, including a new resin infiltration system. Compend Contin Educ Dent. 2009;30 Spec No 3:1-10; quiz 11-2. Review. PubMed PMID: 19894293.

Publications retrieved by additional hand-search: WSL

22

Ogodescu, A, Ogodescu E, Talpoş S, Zetu I. [Resin infiltration of white spot lesions during the fixed orthodontic appliance therapy]. Rev Med Chir Soc Med Nat lasi, 2011;115(4):1251-7. uncontrolled clinical study

masking

24 Paris, S. and H. Meyer-Lueckel, Masking of labial enamel white spot lesions by resin infiltration--a clinical report. In situ, Quintessence Int, 2009;40(9):713-8. bovine

Senestraro, S.V., et al., Minimally invasive resin infiltration of arrested white-spot lesions: A randomized clinical trial. J Am Dent Assoc, 2013;144(9):997-1005.
RCT

Case report 
Table 1. -CONTINUATION- Overview of the articles retrieved by Pubmed, and by additional hand search of references. Of 34 retrieved articles, 29 were included in the review.

29 Tirlet, G., H.F. Chabouis, and J.P. Attal, Infiltration, a new therapy for masking enamel white spots: a 19-month follow-up case series. Eur J Esthet Dent, 2013;8(2):180-90.

Case report

(29) 30 Neuhaus, K.W., et al., Late infiltration of post-orthodontic white spot lesions. J Orofac Orthop, 2010;71(6):442-7.

Case report

31 Hammad, S.M., et al., Effect of resin infiltration on white spot lesions after debonding orthodontic brackets. Am J Dent, 2012;25(1):3-8.

Cohort

(non-control,

prospective)

Publications retrieved by additional hand-search: $\mathrm{MIH}$ and fluorosis

32 Attal, J.P., et al., White spots on enamel: treatment protocol by superficial or deep infiltration (part 2). Int Orthod, 2014;12(1):1-31.
Case report
33 34
Auschill, T.M., K.E. Schmidt, and N.B. Arweiler, Resin Infiltration for Aesthetic Improvement of Mild to Moderate Fluorosis: A Six-month Follow-up Case Report. Oral Health Prev Dent, 2014.
Case report

NR (no infiltration)

\section{References}

1. Heymann, G.C. and D. Grauer, A contemporary review of white spot lesions in orthodontics. J Esthet Restor Dent. 2013. 25(2): 85-95.

2. Julien, K.C., P.H. Buschang, and P.M. Campbell, Prevalence of white spot lesion formation during orthodontic treatment. Angle Orthod. 2013. 83(4): 641-7. 3. Bishara, S.E. and A.W. Ostby, White Spot Lesions: Formation, Prevention, and Treatment. Semin Orthod. 2008, 14(3): 174-182.

4. Ogaard, B., G. Rolla, and J. Arends, Orthodontic appliances and enamel demineralization. Part 1. Lesion development. Am J Orthod Dentofacial Orthop. 1988. 94(1): 68-73.

5. Melrose, C.A., J. Appleton, and B.B. Lovius, A scanning electron microscopic study of early enamel caries formed in vivo beneath orthodontic bands. Br J Orthod. 1996. 23(1): 43-7.

6. Tufekci, E., et al., Prevalence of white spot lesions during orthodontic treatment with fixed appliances. Angle Orthod. 2011. 81(2): 206-10.

7. Richter, A.E., et al., Incidence of caries lesions among patients treated with comprehensive orthodontics. Am J Orthod Dentofacial Orthop. 2011. 139(5): 657-64.

8. Stahl, J. and A.F. Zandona, Rationale and protocol for the treatment of noncavitated smooth surface carious lesions. Gen Dent. 2007. 55(2): 105-11.

9. Paris, S. and H. Meyer-Lueckel, Masking of labial enamel white spot lesions by resin infiltration--a clinical report. Quintessence Int. 2009. 40(9): 713-8.

10. Denbesten, P. and W. Li, Chronic fluoride toxicity: dental fluorosis. Monogr Oral Sci. 2011. 22: 81-96.

11. Elfrink, M.E., et al., Deciduous molar hypomineralization and molar incisor hypomineralization. J Dent Res. 2012. 91(6): 551-5.

12. Lygidakis, N.A., et al., Best Clinical Practice Guidance for clinicians dealing with children presenting with Molar-Incisor-Hypomineralisation $(\mathrm{MIH})$ : An EAPD Policy Document. Eur Arch Paediatr Dent. 2010. 11(2): 75-81.

13. Weerheijm, K.L., B. Jalevik, and S. Alaluusua, Molar-incisor hypomineralisation. Caries Res. 2001. 35(5): 390-1.

14. Borges, A., et al., Color Stability of Resin Used for Caries Infiltration After Exposure to Different Staining Solutions. Oper Dent. 2013.

15. Rocha Gomes Torres, C., et al., Effect of caries infiltration technique and fluoride therapy on the colour masking of white spot lesions. J Dent. 2011. 39(3): 202-7.

16. Yap, J., et al., Evaluation of a novel approach in the prevention of white spot lesions around orthodontic brackets. Aust Dent J. 2014. 59(1): 70-80. 17. Yetkiner, E., et al., Colour improvement and stability of white spot lesions following infiltration, micro-abrasion, or fluoride treatments in vitro. Eur J Orthod. 2014. 36(5): 595-602.
18. Yuan, H., et al., Esthetic comparison of white-spot lesion treatment modalities using spectrometry and fluorescence. Angle Orthod. 2014. 84(2): 343-9.

19. Schwendicke, F., et al., Costs and effectiveness of treatment alternatives for proximal caries lesions. PLoS One. 2014. 9(1): p. e86992. 20. Mueller, J., et al., Surface tridimensional topography analysis of materials and finishing procedures after resinous infiltration of subsurface bovine enamel lesions. Quintessence Int. 2011. 42(2): 135-47.

21. Taher, N.M., Atomic force microscopy and tridimensional topography analysis of human enamel after resinous infiltration and storage in water. Saudi Med J. 2013. 34(4): 408-14.

22. Jia, L., et al., Effect of caries infiltrant application on shear bond strength of different adhesive systems to sound and demineralized enamel. J Adhes Dent. 2012. 14(6):569-74.

23. Torres, C.R., et al., Effect of caries infiltration technique and fluoride therapy on microhardness of enamel carious lesions. Oper Dent. 2012. 37(4): 363-9.

24. Paris, S., et al., Micro-hardness and mineral loss of enamel lesions after infiltration with various resins: influence of infiltrant composition and application frequency in vitro. J Dent. 2013. 41(6): 543-8.

25. Jia, L., et al., Influence of caries infiltrant contamination on shear bond strength of different adhesives to dentin. Clin Oral Investig. 2013. 17(2): 643-8. 26. Wiegand, A., et al., Adhesive performance of a caries infiltrant on sound and demineralised enamel. J Den. 2011. 39(2): 117-21.

27 . Attin, R., et al., Shear bond strength of brackets to demineralize enamel after different pretreatment methods. Angle Orthod. 2012. 82(1): 56-61.

28. Ekizer, A., et al., Effects of demineralizaton-inhibition procedures on the bond strength of brackets bonded to demineralized enamel surface. Korean J Orthod. 2012. 42(1): 17-22.

29. Mews, L., et al., Shear bond strength of orthodontic brackets to enamel after application of a caries infiltrant. Angle Orthod. 2015. 85(4): 645-50.

30. Naidu, E., et al., Shear bond strength of orthodontic resins after caries infiltrant preconditioning. Angle Orthod. 2013. 83(2): 306-12.

31. Paris, S. and H. Meyer-Lueckel, The potential for resin infiltration technique in dental practice. Dent Update. 2012. 39(9): 623-6, 628.

32. Knosel, M., A. Eckstein, and H.J. Helms, Durability of esthetic improvement following Icon resin infiltration of multibracket-induced white spot lesions compared with no therapy over 6 months: a single-center, split-mouth, randomized clinical trial. Am J Orthod Dentofacial Orthop. 2013. 144(1): 86-96. 
33. Meyer-Lueckel, H., S. Paris, and A.M. Kielbassa, Surface layer erosion of natural caries lesions with phosphoric and hydrochloric acid gels in preparation for resin infiltration. Caries Res. 2007. 41(3): 223-30. 34. Ogodescu, A., et al., (Resin infiltration of white spot lesions during the fixed orthodontic appliance therapy). Rev Med Chir Soc Med Nat lasi. 2011. 115(4): 1251-7.

35. Senestraro, S.V., et al., Minimally invasive resin infiltration of arrested white-spot lesions: a randomized clinical trial. J Am Dent Assoc. 2013. 144(9): 997-1005.

36. Paris, S. and H. Meyer-Lueckel, Inhibition of caries progression by resin infiltration in situ. Caries Res. 2010. 44(1): 47-54.

37. Eckstein, A., H.J. Helms, and M. Knosel, Camouflage effects following resin infiltration of postorthodontic white-spot lesions in vivo: One-year follow-up. Angle Orthod. 2015. 85(3): 374-80.

38. Glazer, H.S., Treating white spots: new caries infiltration technique. Dent Today. 2009. 28(10): 82, 84-5.

39. Gugnani, N., et al., Caries infiltration of noncavitated white spot lesions: A novel approach for immediate esthetic improvement. Contemp Clin Dent. 2012. 3(Suppl 2): S199-202.

40. Hammad, S.M., et al., Effect of resin infiltration on white spot lesions after debonding orthodontic brackets. Am J Dent. 2012. 25(1): 3-8.

41. Kim, S., et al., The evaluation of resin infiltration for masking labial enamel white spot lesions. Int J Paediatr Dent. 2011. 21(4): 241-8.

42. Lee, J.H., et al., Minimally invasive treatment for esthetic enhancement of white spot lesion in adjacent tooth. J Adv Prosthodont. 2013. 5(3): 359-63. 43. Neuhaus, K.W., et al., Late infiltration of post-orthodontic white spot lesions. J Orofac Orthop. 2010. 71(6): 442-7.

44. Shivanna, V. and B. Shivakumar, Novel treatment of white spot lesions: A report of two cases. J Conserv Dent. 2011. 14(4): 423-6.

45. Tirlet, G., H.F. Chabouis, and J.P. Attal, Infiltration, a new therapy for masking enamel white spots: a 19-month follow-up case series. Eur J Esthet Dent. 2013. 8(2): 180-90.
46. Feng, C.H. and X.Y. Chu, (Efficacy of one year treatment of icon infiltration resin on post-orthodontic white spots). Beijing Da Xue Xue Bao. 2013. 45(1): 40-3.

47. Borges, A.B., The concept of resin infiltration technique and its multiple applications. J Contemp Dent Pract. 2014. 15(3): p. i.

48. Kielbassa, A.M., J. Muller, and C.R. Gernhardt, Closing the gap between oral hygiene and minimally invasive dentistry: a review on the resin infiltration technique of incipient (proximal) enamel lesions. Quintessence Int. 2009. 40(8): 663-81.

49. Kugel, G., P. Arsenault, and A. Papas, Treatment modalities for caries management, including a new resin infiltration system. Compend Contin Educ Dent. 2009. 30 Spec No 3:1-10; quiz 11-2.

50. Lasfargues, J.J., et al., Minimal intervention dentistry: part 6. Caries inhibition by resin infiltration. Br Dent J. 2013. 214(2): 53-9.

51. Attal, J.P., et al., White spots on enamel: treatment protocol by superficial or deep infiltration (part 2). Int Orthod. 2014. 12(1): 1-31.

52. Auschill, T.M., K.E. Schmidt, and N.B. Arweiler, Resin Infiltration for Aesthetic Improvement of Mild to Moderate Fluorosis: A Six-month Follow-up Case Report. Oral Health Prev Dent. 2015. 13(4): 317-22.

53. Denis, M., et al., White defects on enamel: diagnosis and anatomopathology: two essential factors for proper treatment (part 1). Int Orthod. 2013. 11(2): 139-65.

54. Gugnani, N., et al., Esthetic improvement of white spot lesions and non-pitted fluorosis using resin infiltration technique: series of four clinical cases. J Indian Soc Pedod Prev Dent. 2014. 32(2): 176-80.

55. Munoz, M.A., et al., Alternative esthetic management of fluorosis and hypoplasia stains: blending effect obtained with resin infiltration techniques. J Esthet Restor Dent. 2013. 25(1): 32-9.

56. Torres, C.R. and A.B. Borges, Color masking of developmental enamel defects: a case series. Oper Dent. 2015. 40(1): 25-33. 